\title{
A case of PRES following intracranial hypotension, with spontaneous resolution with epidural blood patch
}

\section{Sarada Murali Mamilla*}

Department of Obstetrics and Gynecology, Yashodha Hospital, Somajiguda, Hyderabad, India

Received: 19 September 2018

Accepted: 12 November 2018

\section{*Correspondence:}

Dr. Sarada Murali Mamilla,

E-mail: mamillasarada@yahoo.co.in

Copyright: (C) the author(s), publisher and licensee Medip Academy. This is an open-access article distributed under the terms of the Creative Commons Attribution Non-Commercial License, which permits unrestricted non-commercial use, distribution, and reproduction in any medium, provided the original work is properly cited.

\begin{abstract}
Posterior reversible encephalopathy syndrome (PRES (also known as reversible posterior leukoencephalopathy syndrome)) presents with rapid onset of symptoms including headache, seizures, altered consciousness, and visual disturbance. It is often but by no means always associated with acute hypertension. Diffusion-weighted MRI is the most sensitive exam to confirm the diagnosis of PRES. If promptly recognized and treated, the clinical syndrome usually resolves within a week, and the changes seen in magnetic resonance imaging (MRI) resolve over days to weeks. Posterior reversible encephalopathy syndrome is an increasingly recognized disorder, with a wide clinical spectrum of both symptoms and triggers, and yet it remains poorly understood. Differential diagnosis includes venous sinus thrombosis, cerebral haemorrhage, encephalitis, vasculititis and metabolic encephalopathys. No clinical trials have evaluated the management of PRES, but rapid withdrawal of the trigger appears to hasten recovery and to avoid complications: for example, aggressive blood pressure management, withdrawal of the offending drug, or delivery in eclampsia. We report a case of women presenting with severe headache, tonic clonic convulsions 7 days after spinal anaesthesia for caesarean delivery, MRI showed evidence of intracranial hypotension and posterior reversible encephalopathy syndrome. She was treated with Blood patch which lead to complete resolution of symptoms and radiological findings. The possible pathogenetic relationship between intracranial hypotension, secondary to the inadvertent dural puncture, and PRES is discussed. Prompt diagnosis and proper treatment results in complete cure without permanent neurological sequelae.
\end{abstract}

Keywords: Neurotoxic, PRES, Spinal headache

\section{INTRODUCTION}

Posterior reversible encephalopathy syndrome (PRES) is a neurotoxic state that occurs secondary to the inability of the posterior circulation to autoregulate in response to acute changes in blood pressure. ${ }^{1}$ The mechanism behind the developing vasogenic edema and CT or MR imaging appearance of PRES is not known. Two theories have historically been proposed:

- Severe hypertension leads to failed auto-regulation, subsequent hyperperfusion, with endothelial injury/vasogenic edema and;
- Vasoconstriction and hypoperfusion leads to brain ischemia and subsequent vasogenic edema. ${ }^{2}$

Commonly, PRES evolves over a matter of hours, with the most common presenting symptoms being seizures, disturbed vision, headache, and altered mental state.

More than $70 \%$ of patients with PRES are hypertensive, though significant proportions have normal or only mildly raised blood pressure. ${ }^{3}$ PRESS is also seen in nonobstetric conditions like solid organ transplantation, infections, sepsis, shock, autoimmune disease, and can also occur following cancer chemotherapy. ${ }^{4}$ 
The differential diagnosis of PRES is broad, Venous sinus thrombosis or subdural, intracerebral, or subarachnoid hemorrhage can all present with headache, seizures, reduced consciousness, and focal neurologic signs. Infective encephalitis or meningitis, particularly herpes simplex encephalitis should be considered, and rapid treatment with intravenous acyclovir and antibiotics may be lifesaving while a diagnosis is still being pursued. It is important to consider the diagnosis of posterior circulation stroke, because its treatment and prognosis both differ from those in PRES. Central nervous system vasculitis can present with symptoms similar to those in PRES, but the MRI findings are usually more diffuse, and many of the clinical features and MRI findings are irreversible. Autoimmune encephalitis and metabolic encephalopathies such as deranged serum glucose, sodium (including central pontine myelinolysis), uremia, or drug toxicity (for example cyclosporine) can also have similar progressive symptoms.

Long term consequences of PRESS have been reported to be cognitive problems, epilepsy, or visual impairment. ${ }^{5}$ Though cases of posterior reversible encephalopathy syndrome (PRES) at term are reported, we tend to overlook this increasingly reported neurologic syndrome. ${ }^{6}$ It is gaining more and more importance as timely diagnosis and proper treatment results in complete recovery without any permanent neurological sequelae. We present a case of PRESS developing secondary to iatrogenic intracranial hypotension and treated with epidural blood patch.

\section{CASE REPORT}

Mrs. K, unbooked case of primi at 37 weeks came with $\mathrm{C} / \mathrm{o}$ of pain in lower abdomen since early morning. After initial assessment she was admitted to LR as she was in early labor. After 8 hours of labor she was taken for emergency caesarean in the view of pathological CTG, delivered alive male baby weight $2.1 \mathrm{~kg}$ with APGAR 2,4 . Baby was shifted to NICU. Day 1 post-partum, she developed headache, more on sitting and standing, diagnosed to have post spinal headache, advised supine position, plenty of liquids and caffeine. Headache worsened. Neurologist opinion taken, CT scan done on day 3 to exclude other causes of headache. Confirmed to have post spinal headache, and was on conservative measures, till Day 6. Patient left against medical advice on day 6 evening and came back to emergency OT with seizures, severe headache, blurring of vision in early hours of Day 7. MRI showed PRES with diffuse meningeal enhancement suggestive of Spontaneous intracranial hypotension. She was started on antiepileptic medicines and supportive measures. No symptomatic improvement noted. Epidural blood patch was planned.10cc blood was drawn from peripheral vein and injected into epidural space at site of previous spinal puncture to resolve spinal leak and patient improved with headache and seizures immediately. Follow up MRI done showed Interval resolution of flair hyper intensities in bilateral cerebellum and basal ganglia. Patient was discharged home and now symptom free.
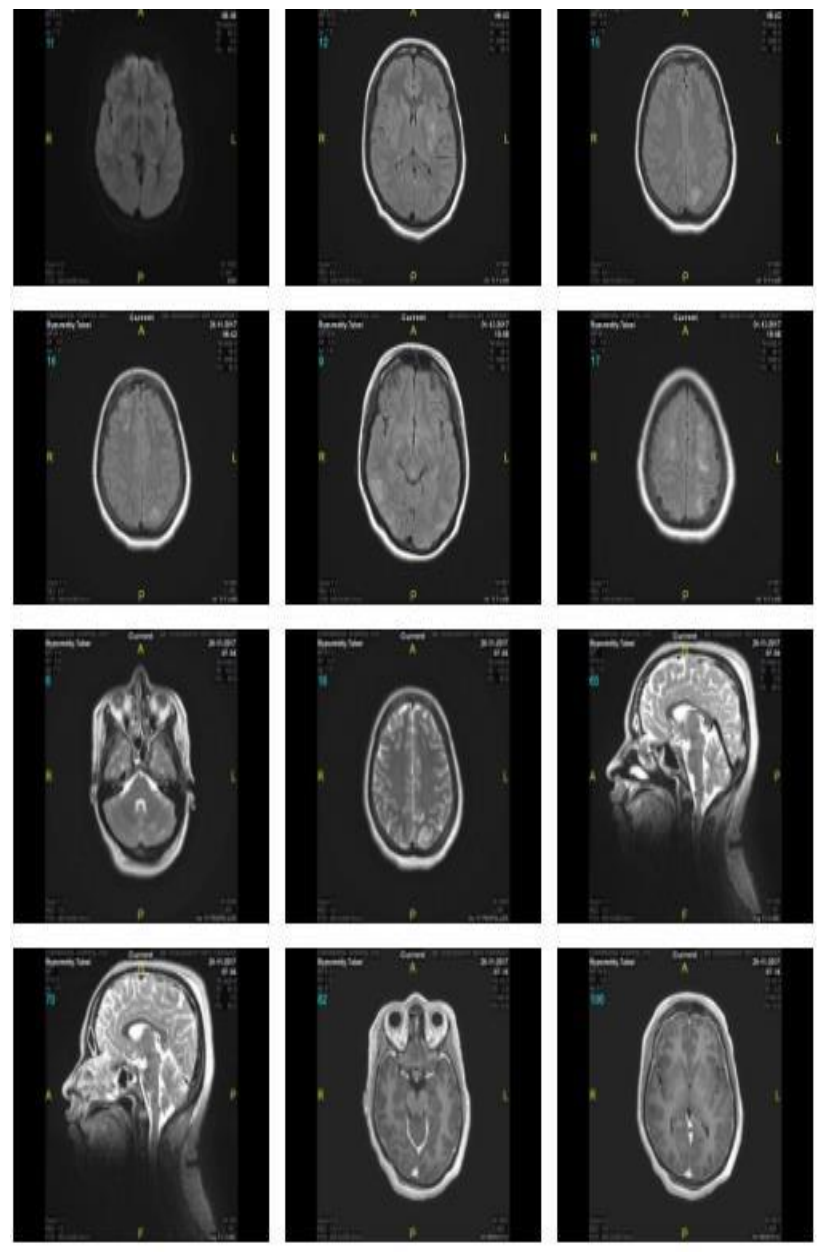

Figure 1: MRI brain- flare intensities in posterior cerebrum.

\section{DISCUSSION}

Post dural puncture headache (PDPH) is a syndrome, clinically characterized by postural headaches elicited or exacerbated by upright position, caused by reduction of CSF volume under normal limits. ${ }^{7}$ This syndrome could be primary (idiopathic) or secondary to a variety of causes and could be the result of inadvertent dural puncture during epidural/spinal anesthesia. (0.26-2\%). ${ }^{7}$ Generally, PDPH is self-limiting and has symptoms subsiding after 2-16 days. Conservative therapy is usually sufficient; nevertheless, in the suspect of a meningeal leak or after a lumbar puncture, epidural blood patch or saline infusion can provide almost immediate relief. Posterior reversible encephalopathy syndrome presents with seizures, disturbed vision, headache and altered mental state. Various clinical settings can precipitate the syndrome. The mechanism is not well understood but is thought to be related to the altered integrity of the blood brain barrier. In our patient PRESS evolved over intracranial hypotension secondary to dural tap. 
Persistent leakage of CSF through the dural opening left by the lumbar puncture needle can result in sagging of the brain and traction on nerves and meningeal vessels.? Presumably, the diminished volume of CSF in the cerebral ventricle may collapse the ventricle. ${ }^{8}$ This traction might cause mechanical stimulation on the arterial wall and, in theory, could induce vasospasm. ${ }^{8}$ In the current patient, diffuse cerebral artery vasospasm, possibly due to the traction of this vessel by anatomic brain displacement, could provide an explanation for the development of PRES after spinal anesthesia.

The goals in the management of PRES include:

- The removal or reduction of the causative drug,

- Management of blood pressure, if hypertension present,

- Control of seizures,

- Magnesium sulphate for cerebral vasospasm and

- Mechanical blockade, epidural blood patch. ${ }^{9}$

In addition, maintenance of airway and arterial oxygenation, sufficient hydration, correction of hypoglycaemia, electrolyte disturbances, or coagulopathy should be taken care of simultaneously.

Other differential diagnoses of convulsions in the peripartum period include intracerebral haemorrhage, thrombotic phenomena, head injury, meningitis, encephalitis, first onset of epilepsy, first manifestation of intracranial tumours or neurocysticercosis. A high index of clinical suspicion in the appropriate situations will help to recognize PRES. Physicians should keep in mind that a postpartum headache could be the clinical manifestation of an underlying intracranial hypotension, which in turn could lead eventually to the development of PRES. Therefore, the importance of correct recognition and diagnosis of PDPH/PRES condition, also using MR imaging, is implicit in order to be able to establish an adequate therapy to prevent possible irreversible ischemic brain damage.

Funding: No funding sources Conflict of interest: None declared

Ethical approval: Not required

\section{REFERENCES}

1. Pugliese S, Finocchi V, Borgia ML, Nania C, Della Vella B, Pierallini A, Bozzao A. Intracranial hypotension and PRES: case report. J Headache Pain. 2010;11(5):437-40.

2. W.S. Bartynski,Posterior Reversible Encephalopathy Syndrome, Part 2: Controversies Surrounding Pathophysiology of Vasogenic Edema,American Journal of Neuroradiol. 2008;29(6):1043-49

3. Santillan A, Aamodt W, Bhavaraju-Sanka R.Pearls and Oy-sters: Spontaneous intracranial hypotension and posterior reversible encephalopathy syndrome. Neurol. 2016;86(6):e55-7.

4. S. Esther V. Hobson, Ian Craven, and S. Catrin Blank, Posterior Reversible Encephalopathy Syndrome: A Truly Treatable Neurologic Illness. Perit Dial Int. 2012;32(6):590-4.

5. Postma IR1, Slager S2, Kremer HP3, de Groot JC4, Zeeman GG, Long-term consequences of the posterior reversible encephalopathy syndrome in eclampsia and preeclampsia: a review of the obstetric and nonobstetric literature. Obstet Gynecol Surv. 2014;69(5):287-300.

6. Achar SK, Shetty N, Joseph TT. Posterior reversible encephalopathy syndrome at term pregnancy. Indian J Anaesth. 2011;55(44):399-401.

7. Ho CM, Chan KH. Posterior Reversible Encephalopathy Syndrome with Vasospasm in a Postpartum Woman After Postdural Puncture Headache Following Spinal Anesthesia. Case report. Anesthes Anal. 2007;105(3):770-2.

8. Mercieri M, Mercieri A, Paolini S, Arcioni R, Lupoi D, Passarelli F. et al. Postpartum cerebral ischaemia after accidental dural puncture and epidural blood patch. Br J Anaesth 2003;90(1):98-100

9. Rajan S, Puthenveettil N, Paul J, Kumar L, Posterior reversible encephalopathy syndrome following caesarean section under spinal anaesthesia, Indian $\mathbf{J}$ Anaesth. 2014;58(6):762-65.

Cite this article as: Mamilla SM. A case of PRES following intracranial hypotension, with spontaneous resolution with epidural blood patch. Int J Reprod Contracept Obstet Gynecol 2018;7:5190-2. 